\title{
Síndrome de Lynch: selección de pacientes para el estudio genético mediante análisis de inestabilidad microsatelital e inmunohistoquímica
}

\author{
ANA MARÍA WIELANDT ${ }^{1}$, ALEJANDRO J. ZÁRATE ${ }^{1}$, \\ CLAUDIA HURTADO ${ }^{1}$, PAULINA ORELLANA ${ }^{1}$, KARIN ÁLVAREZ $^{1}$, \\ ELIANA PINTO $^{1}$, LUIS CONTRERAS ${ }^{2}$, ALEJANDRO CORVALÁN $^{3}$, \\ UDO KRONBERG ${ }^{1}$, FRANCISCO LÓPEZ-KÖSTNER ${ }^{1}$
}

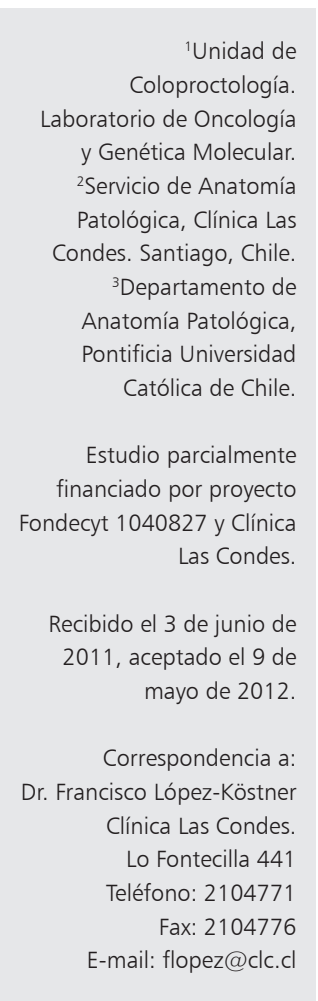

\section{Lynch syndrome: selection of families by micro- satellite instability and immunohistochemistry}

Background: Selection of patients with Lynch Syndrome (LS) for a genetic study involves the application of clinical criteria. To increase the rate of identification of mutations, the use of molecular studies as Microsatellite Instability (MSI) and Immunohistochemistry (IHC) in the tumor has been proposed. Aim: To demonstrate the usefulness of MSI and IHC in the detection of mutations in patients with LS. Material and Methods: From our Familial Colorectal Cancer Registry, families suspected of LS were selected according to Amsterdam or Bethesda clinical criteria. Screening of germline mutations of MLH1, MSH2 and MSH6 genes was performed. In addition, analysis of MSI and IHC were performed in colorectal tumors. Results: A total of 35 families were studied (19 met Amsterdam and 16 met Bethesda criteria). Twenty one families harbored a germline alteration in MLH1, MSH2 or MSH6 (18 Amsterdam and 3 Bethesda). In these families, eighteen different alterations were found, 15 of which were mutations and 3 corresponded to variants of uncertain pathogenicity. On the other hand, $80 \%$ of the tumors showed positive microsatellite instability (27 MSI-high and 1 MSI-low), and immunohistochemical testing showed that 77\% of tumors had the loss of a protein. Correlation between results of tumor molecular studies and the finding of germline nucleotide change showed that IHC and MSI predicted mutations in 81 and 100\% of patients, respectively. Conclusions: MSI and IHC can efficiently select patients with a high probability of carrying a mutation in DNA repair genes.

(Rev Med Chile 2012; 140: 1132-1139).

Key words: Colorectal neoplasms, hereditary non polyposis; Lynch syndrome; Microsatellite instability.
$\mathrm{E}$ 1 cáncer colorrectal (CCR) se encuentra entre las primeras 4 causas de mortalidad en países desarrollados ${ }^{1}$ y en Chile está dentro de las primeras 5 causas tanto en hombres como en mujeres ${ }^{2}$. Se estima que entre 6 y $8 \%$ de los casos de CCR corresponden a un síndrome hereditario ${ }^{3}$, el más común de estos síndromes es el cáncer colorrectal hereditario no poliposo (CCHNP) o síndrome de Lynch $(\mathrm{SL})^{4}$.
El SL es una enfermedad con herencia autosómica dominante, que se caracteriza por un riesgo aumentado de desarrollo de CCR a edades más tempranas y de localización principalmente proximal (colon derecho), en comparación con los casos de CCR esporádico. Además, este síndrome se caracteriza por un riesgo aumentado de desarrollo de otros cánceres en diversos órganos, tales como: endometrio, ovario, estómago, intestino delgado, 
urotelio, cerebro y piel ${ }^{4,5}$. Por lo tanto, el definir si una persona es portadora de este síndrome es determinante para optimizar el manejo médico y seguimiento clínico. La identificación de familias sospechosas de SL se realiza a través de criterios clínicos, conocidos como Amsterdam ${ }^{6}$ y Bethes$\mathrm{da}^{7}$ (Tabla 1). Este síndrome se origina por una mutación en uno de los genes que codifican para enzimas que participan en el sistema de reparación de errores del $\mathrm{ADN}^{8}$. Los genes MLH1 o MSH2 se encuentran mutados en $90 \%$ de los casos con SL, en tanto que otros genes como MSH6 y PMS2 podrían dar cuenta del resto de las mutaciones ${ }^{4}$.

Uno de los marcadores moleculares que ha sido utilizado en el SL para revelar de forma indirecta la probable presencia de mutaciones en la línea germinal, es el estudio de inestabilidad microsatelital (MSI) en el tejido tumoral colorrectal ${ }^{9,10}$. Los microsatélites son regiones del ADN con múltiples repeticiones de uno a ocho nucleótidos (por ejemplo, AAAAA o CGCGCGCG). Estos microsatélites son particularmente susceptibles a errores durante el proceso de replicación del ADN, los cuales son corregidos por un sistema de reparación, sin embargo, cuando este sistema se encuentra dañado, estos errores permanecerán ${ }^{11}$, lo que se reflejará en un aumento o disminución de la longitud de los microsatélites en el tejido tumoral comparado con el tejido normal ${ }^{12,13}$.

Otro marcador molecular sugerido para la pesquisa de pacientes con SL, es la detección de la expresión de las proteínas involucradas en el sistema de reparación del $\mathrm{ADN}$ mediante la técnica de inmunohistoquímica (IHQ). Generalmente una mutación en la línea germinal conlleva una pérdida de expresión de la proteína en el tejido tumoral, ya sea por degradación de ésta o porque la proteína expresada (trunca) no es detectable por los anticuerpos utilizados ${ }^{14}$.

El objetivo de este estudio es evaluar la utilidad de la MSI y la IHQ para tamizar a pacientes con sospecha clínica de SL y así definir la mejor estrategia de estudio aplicable a este grupo de pacientes, en un país con recursos limitados como Chile.

\section{Materiales y Métodos}

\section{Pacientes}

Se seleccionaron los pacientes índices de $35 \mathrm{fa}$ milias del Registro de Cáncer Colorrectal Familiar, reclutados desde el año $2003 \mathrm{al}$ 2010, provenientes
Tabla 1. Criterios de selección de familias sospechosas de Síndrome de Lynch (CCHNP)

Amsterdam I y II

- Tres familiares con cáncer de colon o cánceres asociados a CCHNP, uno de ellos debe ser pariente en primer grado de los otros dos

- Dos generaciones sucesivas afectadas

- Al menos un caso diagnosticado antes de los 50 años

Criterios de Bethesda

- Persona con 2 tipos de cáncer asociados a CCHNP

- Una persona con cáncer de colon y un pariente en primer grado con cáncer de colon y/o cánceres asociados a CCHNP ( $<44$ años) y/o adenomas ( $<40$ años)

- Persona con cáncer de colon o endometrio antes de los 45 años

- Persona con cáncer de colon en lado derecho que presenten bajo grado de diferenciación antes de los 45 años

de diferentes lugares del país. Las familias fueron clasificadas según el cumplimiento de los criterios clínicos de Amsterdam o Bethesda. Todos los pacientes firmaron consentimiento informado.

\section{Extracción de ADN}

La extracción de ADN genómico para el análisis mutacional de la línea germinal se realizó a partir de una muestra de sangre periférica utilizando el método descrito por Lahiri y Nurnberger ${ }^{15}$. Del mismo paciente, el ADN tumoral fue extraído a partir de tejido fijado en formalina y embebido en parafina. El tejido tumoral fue reconocido en secciones teñidas con hematoxilina-eosina. Luego, desde cortes seriados de $8 \mathrm{~m}$ de espesor no teñidos, se obtuvieron las células tumorales a partir de las cuales se extrajo el ADN mediante el kit de extracción QIAmp DNA FFPE Tissue de QIAGEN según instrucciones de manufactura.

\section{Análisis de mutaciones y rearreglos genómicos en los genes MLH1, MSH2 y MSH6}

La identificación de mutaciones en los genes MLH1, MSH2 y MSH6 fue realizada mediante amplificación por PCR seguido del análisis de SSCP (Single Strand Conformation Polymorphisms). Todos los partidores utilizados fueron previamente descritos $^{16,17}$. Pacientes sin mutación identificada mediante esta metodología fueron analizados para la búsqueda de grandes rearreglos genómicos en 
los genes MLH1 y MSH2 utilizando la técnica de MLPA (Multiplex Ligation-dependent Probe Amplification), con el kit SALSA P003 (MRC-Holland) que analiza los genes MLH1 y MSH2. El análisis fue realizado de acuerdo a las instrucciones de manufactura $^{18}$.

\section{Análisis de MSI}

El análisis de inestabilidad microsatelital se determinó mediante la amplificación por PCR de los cinco marcadores microsatelitales recomendados por el National Cancer Institute (NCI) para el cáncer colorrectal: los mononucléotidos Bat25, Bat-26 y los dinucleótidos D2S123, D3S1029, D5S346 ${ }^{19,20}$. Cada marcador microsatelital fue amplificado a partir de ADN tumoral y ADN control obtenido a partir de sangre periférica del mismo paciente utilizando partidores marcados con fluoróforos según lo descrito ${ }^{21-23}$. La reacción de PCR fue realizada con 40 ciclos a $55^{\circ} \mathrm{C}$ en un volumen total de $25 \mu$ l. La mezcla de reacción contiene: Buffer $1 \mathrm{X}$ PCR $(50 \mathrm{mM} \mathrm{KCl}, 20 \mathrm{mM}$ Tris $\mathrm{HCl}$ $\mathrm{pH} 8,3), 1,5 \mathrm{mM} \mathrm{MgCl} 2,0,2 \mathrm{mM}$ de cada partidor 0,2 mM de cada dNTP, y 0,6 u de Polimerasa Taq Platinum (Invitrogen). Los microsatélites amplificados fueron separados por electroforesis capilar mediante el secuenciador ABI 310. Los resultados de la separación en el secuenciador se obtuvieron mediante el software Gene Mapper 4.0 de Applied Biosystems. Los tumores que presentan 2 o más microsatélites $(>30 \%)$ con un número de alelos aumentado (inestables) y/o alteración en el patrón de migración son considerados como MSI-alto, tumores con sólo un microsatélite inestable son considerados como MSI-bajo y los tumores que no presentan microsatélites inestables se consideran como estables (MSS) ${ }^{19}$.

\section{Análisis de inmunohistoquímica}

La evaluación de la expresión de las proteínas MLH1, MSH2 y MSH6 en el tumor fue realizada por IHQ en secciones de tejido fijado en formalina y embebido en parafina. El análisis IHQ fue realizado en el laboratorio de anatomía patológica de la Pontificia Universidad Católica de Chile para los pacientes reclutados entre el 2003 y el 2007 y en Clínica Las Condes para aquellos desde el 2008 al 2010. Cortes histológicos fueron montados en portaobjetos con carga positiva y desparafinados. Los pacientes procesados durante el período del 2003 al 2007 fueron evaluados utilizando los anticuerpos para MLH1 (policlonal Ab-2,cat
\#PC56) y MSH2 (Monoclonal Ab-2 cat \#NA27) de Oncogene Research Products según lo descrito previamente $^{15}$ y durante los años 2008 al 2010 se utilizaron anticuerpos de Dako (clone ES05) Novocastra (clone 25D12, dilución 1:50) y Cell Marque (clone 44, dilución 1:50) para MLH1, MSH2 y MSH6 respectivamente. Brevemente, la recuperación de la inmunoreactividad en PT link (Dako) se realizó durante $20 \mathrm{~min}$ a $95^{\circ} \mathrm{C}$ en una solución de alto pH del kit EnVision (Dako). La tinción IHQ se realizó de forma automatizada en el Autostainer 48 (Dako) usando el kit EnVision (Dako) según las instrucciones del fabricante. Para el contraste nuclear se utilizó hematoxilina de Mayer. Se consideró como tinción negativa o ausencia de la expresión de la proteína cuando menos del 10\% de las células tumorales demostraron un patrón de tinción nuclear.

\section{Resultados}

En este estudio fueron analizadas un total de 35 familias sospechosas de SL, 19 cumplían con los criterios Amsterdam y 16 cumplían con los criterios Bethesda. Los estudios genéticos revelaron que 21 familias sospechosas del SL (18 Amsterdam y 3 Bethesda) portaban un cambio nucleotídico, con una tasa de detección de 60\% (18/19, 95\% para familias Amsterdam y 3/16, 19\% en familias Bethesda). Las alteraciones fueron localizadas principalmente en el gen MLH1 (14/21,67\%), seguido del gen MSH2 (5/21, 24\%) y del gen MSH6 (2/21, $9 \%)$. Se observó un efecto patogénico debido a pérdida de dominios funcionales importantes en 18/21 alteraciones, en tanto que 3 cambios correspondieron a variantes de patogenicidad incierta (familias 2, 21 y 28) (Tabla 2).

$\mathrm{El}$ análisis de MSI en los tumores colorrectales reveló que 28 de las 35 familias (80\%) presentaban inestabilidad microsatelital, 27 fueron MSI-alto y 1 fue MSI-bajo, en 75\% de ellas (21/28) se identificó una mutación. Los tumores con MSI fueron principalmente encontrados en familias que cumplen con los criterios de Amsterdam (95\% comparado con $62,5 \%$ de las familias Bethesda) (Tabla 3). El único tumor con MSI-bajo corresponde a un paciente Bethesda sin mutación identificada. La correlación de los resultados de MSI con el hallazgo del estudio genético de la línea germinal demostró que $100 \%$ de los pacientes con mutación o variante alélica identificada, presentan MSI-alta. 
Síndrome de Lynch: selección de pacientes para el estudio genético - A. M. Wielandt et al

Tabla 2. Análisis de inestabilidad microsatelital e inmunohistoquímica en el tejido tumoral de familias con estudio de mutaciones en la línea germinal

\begin{tabular}{|c|c|c|c|c|c|}
\hline $\begin{array}{c}\mathbf{N}^{\circ} \\
\text { Familia }\end{array}$ & $\begin{array}{l}\text { Criterio de } \\
\text { selección }\end{array}$ & Mutación & $\begin{array}{l}\text { Gen } \\
\text { mutado }\end{array}$ & MSI & $\begin{array}{l}\text { IHQ } \\
\text { MLH1-MSH2-MSH6 }\end{array}$ \\
\hline 1 & Ámsterdam & c. $1731+3$ A>T exón 15 & MLH1 & MSI-alta & MLH1 ausente \\
\hline 2 & Ámsterdam & c. 677 + 5 G>A exón 8 & $\mathrm{MLH} 1 *$ & MSI-alta & MLH1 ausente \\
\hline 3 & Bethesda & sin mutación & sin mutación & MSI-alta & MSH6 ausente \\
\hline 4 & Ámsterdam & deleción exón 19 & MLH1 & MSI-alta & MSH2 ausente \\
\hline 5 & Bethesda & sin mutación & sin mutación & MSI-estable & presentes \\
\hline 6 & Ámsterdam & c.503 dupA exón 6 & MLH1 & MSI-alta & MLH1 ausente \\
\hline 8 & Ámsterdam & deleción exón 1 & MLH1 & MSI-alta & MLH1/MSH6 ausentes \\
\hline 9 & Ámsterdam & c.901 C>T exón 11 & MLH1 & MSI-alta & MLH1 ausente \\
\hline 10 & Ámsterdam & deleción exón 19 & MLH1 & MSI-alta & MLH1 ausente \\
\hline 11 & Bethesda & c.2041 G>A exón 18 & MLH1 & MSI-alta & presentes \\
\hline 12 & Ámsterdam & c.2185-2192 del(7) ins.CCCT exón 13 & $\mathrm{MSH} 2$ & MSI-alta & MLH1/MSH2 ausentes \\
\hline 14 & Bethesda & sin mutación & sin mutación & MSI-alta & MLH1 ausente \\
\hline 16 & Bethesda & sin mutación & sin mutación & MSI-alta & presentes \\
\hline 17 & Bethesda & sin mutación & sin mutación & MSI-estable & presentes \\
\hline 18 & Ámsterdam & deleción exones 14-15 & MLH1 & MSI-alta & MLH1 ausente \\
\hline 19 & Bethesda & c.2092-2093 delTC exón 18 & MLH1 & MSI-alta & MLH1 ausente \\
\hline 20 & Bethesda & sin mutación & sin mutación & MSI-baja & presentes \\
\hline 21 & Ámsterdam & c.359 $\mathrm{T}>\mathrm{C}$ & MSH6* & MSI-alta & MLH1 ausente \\
\hline 24 & Bethesda & sin mutación & sin mutación & MSI-alta & MLH1 ausente \\
\hline 25 & Bethesda & sin mutación & sin mutación & MSI-estable & presentes \\
\hline 26 & Ámsterdam & sin mutación & sin mutación & MSI-estable & MLH1 ausente \\
\hline 27 & Ámsterdam & c.1215 C>A exón 7 & $\mathrm{MSH} 2$ & MSI-alta & MLH1 ausente \\
\hline 28 & Ámsterdam & c.26dup GATT 3'UTR & MSH6* & MSI-alta & MSH2/MSH6 ausentes \\
\hline 29 & Ámsterdam & c.790 + 1 G>A exón 9 & MLH1 & MSI-alta & MLH1 ausente \\
\hline 30 & Bethesda & sin mutación & sin mutación & MSI-alta & MLH1 ausente \\
\hline 31 & Ámsterdam & c.2038 C>T exón 13 & $\mathrm{MSH} 2$ & MSI-alta & MSH2 ausente \\
\hline 35 & Bethesda & sin mutación & sin mutación & MSI-estable & MLH1 ausente \\
\hline 36 & Bethesda & sin mutación & sin mutación & MSI-estable & presentes \\
\hline 37 & Ámsterdam & c.1038 + 1 G>T exón 11 & MLH1 & MSI-alta & MLH1 ausente \\
\hline 39 & Bethesda & sin mutación & sin mutación & MSI-alta & MLH1 ausente \\
\hline 44 & Bethesda & sin mutación & sin mutación & MSI-estable & presentes \\
\hline 47 & Ámsterdam & deleción exón 2 & $\mathrm{MSH} 2$ & MSI-alta & MSH2/MSH6 ausentes \\
\hline 56 & Ámsterdam & deleción exón 1 & MLH1 & MSI-alta & MLH1 ausente \\
\hline 57 & Ámsterdam & c.2041 G>A exón 18 & MLH1 & MSI-alta & MLH1 ausente \\
\hline 100 & Bethesda & c.897 T>G exón 5 & $\mathrm{MSH} 2$ & MSI-alta & MSH2 ausente \\
\hline
\end{tabular}

*Variantes alélicas de patogenicidad incierta. 
Síndrome de Lynch: selección de pacientes para el estudio genético - A. M. Wielandt et al

Tabla 3. Familias sospechosas de Síndrome de Lynch según criterios clínicos, resultados de la MSI en el tumor y presencia de mutación en línea germinal

\begin{tabular}{|c|c|c|c|c|}
\hline Criterio clínico & $\begin{array}{c}N^{\circ} \text { de } \\
\text { familias }\end{array}$ & Fenotipo MSI & $\mathbf{N}$ & $\begin{array}{c}\text { Presencia de } \\
\text { mutación }\end{array}$ \\
\hline \multirow[t]{2}{*}{ Amsterdam } & 19 & MSI-alta & $18(95 \%)$ & $18(100 \%)$ \\
\hline & & MSS & $1 \quad(5 \%)$ & $0 \quad(0 \%)$ \\
\hline \multirow[t]{2}{*}{ Bethesda } & 16 & MSI-alta/baja & $10(62,5 \%)$ & $3(30 \%)$ \\
\hline & & MSS & $6(37,5 \%)$ & $0 \quad(0 \%)$ \\
\hline Total & 35 & MSI-alta & $28(80 \%)$ & $21 \quad(75 \%)$ \\
\hline
\end{tabular}

MSI: inestabilidad microsatelital, MSS: estabilidad microsatelital.

Tabla 4. Familias sospechosas de Síndrome de Lynch según criterios clínicos, resultados de la IHQ en el tumor y presencia de mutación en línea germinal

\begin{tabular}{|lccc|}
\hline Criterio clínico & $\begin{array}{c}\mathbf{N}^{\circ} \text { de } \\
\text { familias }\end{array}$ & $\begin{array}{c}\text { IHQ } \\
\text { Ausencia de proteína }\end{array}$ & $\begin{array}{c}\text { Correlación } \\
\text { IHQ con mutación }\end{array}$ \\
\hline Amsterdam & 19 & $19(100 \%)$ & $15(79 \%)$ \\
Bethesda & 16 & $8(50 \%)$ & $2(25 \%)$ \\
Total & 35 & $27(77 \%)$ & $17(63 \%)$ \\
\hline
\end{tabular}

IHQ: inmunohistoquímica.

El análisis de IHQ demostró la pérdida de expresión en el tejido tumoral de al menos una de las proteínas en 27 pacientes $(77 \%)$ y en el 63\% (17/27) de ellas se identificó una mutación. De los 27 pacientes con pérdida de la expresión de la proteína, 19 tuvieron pérdida de MLH1, 3 de MSH2 y 1 de MSH6, y en 4 casos se observó la pérdida de más de una proteína (2 casos MSH2 y MSH6, 1 caso MLH1 y MSH2, 1 caso MLH1 y MSH6) (Tablas 2 y 4).

La correlación de los resultados de IHQ con el hallazgo de mutación de la línea germinal demostró que $17 / 21(81 \%)$ pacientes tienen pérdida de expresión de la proteína cuyo gen es portador de una mutación o variante alélica. En cuatro casos no observamos esta correspondencia ya sea porque no se observó la pérdida de las proteínas (familia 11) o bien porque la proteína perdida no correspondía al gen mutado (familias 4, 21 y 27) (Tabla 2).

\section{Discusión}

Se ha establecido que las familias candidatas a estudios genéticos deben ser preseleccionadas mediante su historia familiar, análisis de IHQ y de MSI para aumentar el éxito en el hallazgo de mutaciones en los pacientes con SL ahorrando tiempo y costo en los estudios ${ }^{24-26}$. Una de las limitaciones respecto de los antecedentes familiares, es que en ocasiones la información que expresan los familiares no es exacta.

Los resultados de nuestro trabajo demuestran que el criterio de selección más estricto (Amsterdam) presentó una mayor probabilidad de identificación de alteraciones en la línea germinal al comparar con la utilización del criterio Bethesda (95\% versus 19\%). Destacamos que la única familia Amsterdam (familia 26) que no presenta inestabilidad en los microsatélites (MSS) podría corresponder a un Síndrome Familiar Colorrectal tipo $\mathrm{X}$, cuya causa genética no ha sido identificada a la fecha ${ }^{27,28}$.

La correlación de este estudio con los criterios clínicos demostró que $95 \%$ de las familias que cumplen con los criterios de Amsterdam (18/19) tiene MSI alta y sólo 62,5\% (10/16) de las familias Bethesda presentan tumores con MSI. Si el estudio genético hubiera sido realizado sólo en este grupo de pacientes con tumores con MSI, el porcentaje de identificación de alteraciones en la línea germinal habría aumentado de 95\% (18/19) a 100\% (18/18) 
en las familias Amsterdam y de 19\% (3/16) a 30\% (3/10) en las familias Bethesda.

La correlación de los resultados de IHQ con los criterios clínicos demostró que 79\% (15/19) de las familias Amsterdam sufren la pérdida de la expresión de la proteína cuyo gen presenta una mutación, mientras que sólo en 25\% (2/8) de las familias Bethesda se observó esta correlación. Esta observación confirma la utilización del criterio clínico de Amsterdam como primera opción de tamizaje.

En los casos de las familias 4 y 11, las mutaciones identificadas se encuentran localizadas al final del gen MLH1 (deleción del exón 19 y un cambio aminoacídico en el exón 18, respectivamente), sin embargo, no se observa ausencia de la proteína correspondiente. Esto se puede explicar ya que la proteína puede estar presente, pero no necesariamente ser funcional.

En el caso de las familias 21 y 27 los cambios se identificaron en el exón 2 del gen MSH6 (un cambio aminoacídico de patogenicidad incierta) y en el exón 7 del gen MSH2 (una proteína con término prematuro), respectivamente; sin embargo, en ambos casos, la IHQ demostró ausencia sólo de MLH1. La detección de las respectivas proteínas por IHQ se puede deber a que en ambos casos, se mantuvo el sitio de reconocimiento de los anticuerpos, y la ausencia de MLH1 observada se puede deber a una alteración de la antigenicidad de la proteína en los tumores debido al tiempo de fijación en formalina como se ha propuesto en otros trabajos ${ }^{29-31}$ ya que estos provienen de distintos centros del país con distintos protocolos de fijación.

Se ha descrito que otros factores que pueden afectar los resultados de la IHQ mostrando discrepancia entre el hallazgo de mutación y la pérdida de la proteína, pueden explicarse por epimutaciones constitucionales del gen MLH1 o MSH2 dando cuenta de un tercio de los casos de familias que cumplen con los criterios clínicos de CCHNP sin mutación identificada ${ }^{32,33}$ o por hipermetilación del promotor del gen MLH1 en el tumor, que silencia la expresión de la proteína como podría ser el caso de las familias 14, 24, 30 y $39^{34,35}$. También la pérdida de más de una proteína por pérdida del compañero de interacción MSH2/ MSH6 como son los casos de las familias 28 y 47 y la falta de evaluación de otros genes reparadores como PMS2. Por otra parte, las técnicas de SSCP y MLPA utilizadas no evalúan el promotor de los genes estudiados ni regiones alejadas de los sitios de unión intrón-exón ni la presencia de grandes deleciones en el gen MSH6 de manera que pueden quedar alteraciones sin identificar. Otra de las limitaciones del presente estudio es que este se extendió por un período prolongado de tiempo con un cambio de anticuerpos durante el estudio, lo que podría haber influido en los resultados de la IHQ. No obstante, a todos los pacientes se les realizó el estudio genético independiente de su resultado inmunohistoquímico.

En este trabajo, el estudio de MSI presenta una ventaja por sobre la IHQ, proporcionando información sobre la función de las proteínas independiente de su expresión. Sin embargo, no se evaluó el gen PMS2 que podría generar alta inestabilidad por ser una de las proteínas involucradas en el complejo de reparación del ADN, y podría explicar algunos casos en los cuales no se encontró mutación en los genes MLH1, MSH2 y MSH6. El incluir este gen dentro de los estudios genéticos y su determinación por IHQ podría aumentar el éxito de hallazgo de mutaciones y su correlación con el análisis IHQ.

Finalmente, los estudios genéticos para enfermedades hereditarias no se encuentran ampliamente disponibles e implican la utilización de recursos que son limitados. De acuerdo a nuestros resultados tanto la MSI como la IHQ son herramientas que ayudan al hallazgo de mutaciones en pacientes con sospecha de Síndrome de Lynch. Los estudios de MSI y de IHQ pueden seleccionar eficientemente pacientes con alta probabilidad de ser portadores de una mutación en los genes reparadores del ADN y conducir el estudio genético en familias con escasa historia familiar. Proponemos que en los pacientes que cumplen con los criterios de Amsterdam, se puede realizar el estudio genético directamente, evitando la realización de estudios moleculares en el tumor e iniciar los estudios por el gen MLH1 según se muestra en el algoritmo (Figura 1). Por otra parte, para aumentar la probabilidad de identificar una mutación en pacientes Bethesda, es necesario realizar el análisis de MSI e IHQ para seleccionar pacientes que requieran estudio genético y dirigir los estudios al gen candidato optimizando recursos y gastos en pacientes con baja probabilidad de éxito en el hallazgo de mutaciones. 


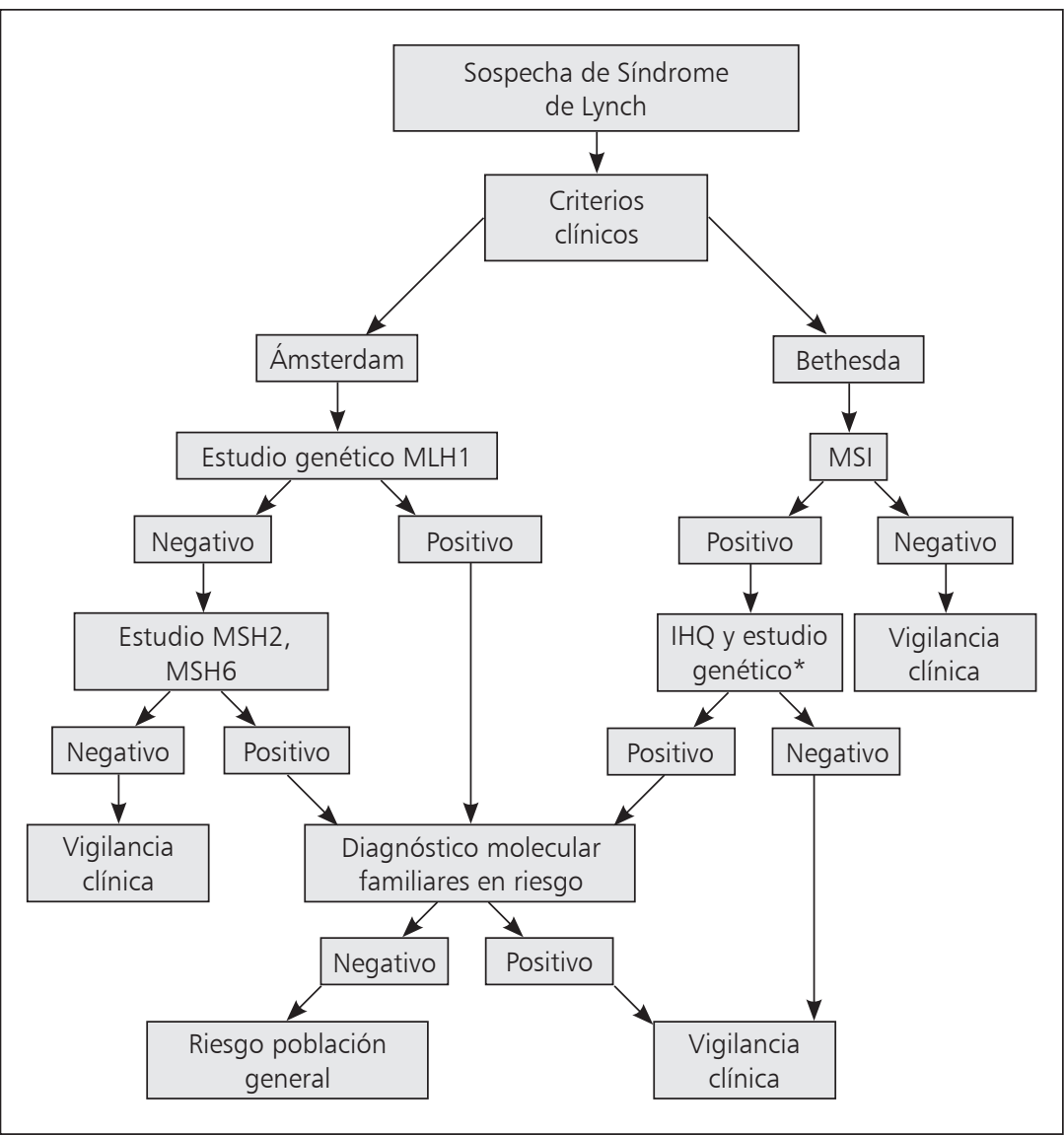

Figura 1. Algoritmo para estudio genético de pacientes con sospecha de Síndrome de Lynch. MSI: inestabilidad microsatelital. IHQ: inmunohistoquímica. *Estudio según resultado de la IHQ u orden establecido para pacientes Ámsterdam.

\section{Referencias}

1. Jemal A, Bray F, Center MM, Ferlay J, Eard E, Forman D. Global ancer statistics. CA Cancer J Clin 2011; 61: 69-90.

2. Disponible en la página web: http://deis.minsal.cl/index. asp.

3. Strate L, Syngal S. Hereditary colorectal cancer syndromes. Cancer Causes Control 2005; 16: 201-13.

4. Lynch $\mathrm{H}$, de la Chapelle A. Hereditary colorectal cancer. N Engl J Med 2003; 348: 919-32.

5. Aarnio M, Sankila R, Pukkala E, Salovaara R, Aaltonen LA, de la Chapelle A, et al. Cancer risk in mutation carriers of DNA mismatch-repair genes. Int J Cancer 1999; 81:214-8.

6. Vasen HF, Watson P, Mecklin JP, Lynch HT. New clinical criteria for hereditary nonpolyposis colorectal cancer (HNPCC, Lynch Syndrome) proposed by the international collaborative group on HNPCC. Gastroenterology 1999; 116: 1453-6.
7. Rodríguez-Bigas MA, Boland CR, Hamilton SR, Henson DE, Jass JR, Khan PM, et al. A National Cancer Institute Workshop on Hereditary Nonpolyposis Colorectal Cancer Syndrome: meeting highlights and Bethesda guidelines. J Natl Cancer Inst 1997; 89: 1758-62.

8. Peltomaki P, Vasen HF. Mutations predisposing to hereditary nonpolyposis colorectal cancer: database and results of a collaborative study. The International Collaborative Group on Hereditary Nonpolyposis Colorectal Cancer. Gastroenterology 1997; 113: 1146-58.

9. Zhang L. Immunohistochemistry versus Microsatellite Instability Testing for screening Colorectal Cancer patients at risk for Hereditary Nonpolyposis Colorectal Cancer Syndrome. Part II. The utility of MSI. J Mol Diagn 2008, 10: 301-7.

10. Douillard JY. Microsatellite instability and mismatch repair genes in colorectal cancer: useful tools for managing patients and counseling their relatives. Clin Colorectal Cancer 2010; 9: 193-4.

11. Boland CR, Goel A. Microsatellite instability in colorec- 
tal cancer. Gastroenterology 2010; 138: 2073-87.

12. Söreide K, Janssen EA, Söiland H, Körner H, Baak JP. Microsatellite instability in colorectal cancer. Br J Surg 2006; 93: 395-406.

13. Thibodeau SN, French A], Cunningham JM, Tester D Burgart L, Roche PQ, et al. Microsatellite instability in colorectal cancer: different mutator phenotypes and the principal involvement of hMLH1. Cancer Res 1998; 58: 1713-8.

14. Shia J. Immunohistochemistry versus microsatellite instability testing for screening colorectal cancer patients at risk for hereditary nonpolyposis colorectal cancer syndrome. Part I. The utility of Immunohistochemistry. J Mol diagn 2008; 10: 301-7.

15. Lahiri DK, Nurnberger JI Jr. A rapid non-enzymatic method for the preparation of HMW DNA from blood for RFLP studies. Nucleic Acids Res 1991; 19: 5444.

16. Wu Y, Berends M, Mensink RG, Kempinga O, Sijmons RH, van Der Zee AG, et al. Association of hereditary nonpolyposis colorectal cancer-related tumors displaying low microsatellite instability with MSH6 germline mutations. Am J Hum Genet 1999; 65: 1291-8.

17. Kolodner RD, Tytell JD, Schmeits J, Kane MP, Gupta RD, Weger, et al. Germ-line msh6 mutations in colorectal cancer families. Cancer Res 1999; 59: 5068-74.

18. Schouten J月, McElgunn C, Waaijer R Zwijnenburg D, Diepvens 3, Pals G Relative quantification of 40 nucleic acid sequences by multiplex ligation-dependent probe amplification. Nucleic Acids Res 2002; 30: e57.

19. Boland CR, Thibodeau SN, Hamilton SR, Sidransky D, Eshleman JR, Burt RW, et al. A National Cancer Institute Workshop on Microsatellite Instability for cancer detection and familial predisposition: development of international criteria for the determination of microsatellite instability in colorectal cancer. Cancer Res 1998; 58: 5248-57.

20. Umar A, Boland CR, Terdiman JA, Syngal S, de la Chapelle A Rüschoff, et al. Revised Bethesda Guidelines for hereditary nonpolyposis colorectal cancer (Lynch syndrome) and microsatellite instability. DNatl Cancen Inst 2004; 96: 261-8.

21. Zárate A Á varez K, Wielandt AM, Hevia M, De la Fuente M, Carvallo 1 , et al. Hereditary non-polyposis colorectal cancer. Report of four siblings. Rev Med Chile 2008; 136: 757-62.

22. Loukola A, Eklin K, Laiho P, Salovaara R, Kristo P, Jarvinen $\mathrm{H}$, et al. Microsatellite marker analysis in screening for hereditary nonpolyposis colorectal cancer (HNPCC). Cancer Res 2001; 61: 4545-9.

23. Raptis S, Mrkonjic M, Green RC, Pethe VV, Monga N,
Chan YM, et al. MLH1 -93G>A promoter polymorphism and the risk of microsatellite-unstable colorectal cancer, J Natl Cancer Inst 2007; 99: 463-74.

24. Aaltonen LA Salovaara R, Kristo Canzian H, Hemmin ki A, Peltomäki $\mathrm{H}$ et al. Incidence of hereditary nonpolyposis colorectal cancer and the feasibility of molecular screening for the disease. Engl J Med 1998; 338: 1481-7.

25. Kurzawski G, Suchy J, Debniak T, Kładny J, Lubi ski J. Importance of microsatellite instability (MSI) in colorectal cancer: MSI as a diagnostic tool. Ann Oncol 2004; 15: iv283-4.

26. De la Chapelle A Hampel H. Clinical relevance of microsatellite instability in colorectal cancer. [Clin Onco] 2010; 28: 3380-7.

27. Lindor NM. Familial colorectal cancer type X: the other half of hereditary nonpolyposis colon cancer syndrome. Surg Oncol Clin N Am 2009 Oct; 18 (4): 637-45.

28. Chen HM, Fang JY. Genetics of the hamartomatous polyposis syndromes: a molecular review. Int J Colorecta] Dis 2009; 24: 865-74.

29. Woods MO, Younghusband HB, Parfrey PS, Gallinger S, McLaughlin Dicks B et al. The genetic basis of colorectal cancer in a population-based incident cohort with a high rate of familial disease. Gut 2010; 59: 1369-77.

30. Overbeek L1, Ligtenberg M), Willems RW, Hermens R, Blokx WA Dubois Sy, et al. Interpretation of immunohistochemistry for mismatch repair proteins is only reliable in a specialized setting. Am J Surg Patho 2008; 32: 1246-51.

31. Chapusot C, Martin L, Puig PL, Ponnelle T, Cheynel N, Bouvier AM, et al. What is the best way to assess microsatellite instability status in colorectal cancer? Study on a population base of 462 colorectal cancers. Am J Surg Pathol 2004; 28: 1553-9.

32. Hitchins MH Ward RD Constitutional (germline) MLH1 epimutation as an aetiological mechanism for hereditary non-polyposis colorectal cancer. IMed Genet 2009; 46: 793-802.

33. Kuiper RA, Vissers LA, Venkatachalam R, Bodmer D, Hoenselaar $\mathrm{E}$, Goossens M, et al. Recurrence and variability of germline EPCAM deletions in Lynch syndrome. Hum Mutat 2011; 32: 407-14.

34. Gazzoli 1, Loda M, Garber, Syngal S, Kolodner RD. A hereditary nonpolyposis colorectal carcinoma case associated with hypermethylation of the MLH1 gene in normal tissue and loss of heterozygosity of the unmethylated allele in the resulting microsatellite instabilityhigh tumor. Cancer Res 2002; 62: 3925-8.

35. Oda S, Zhao Y Maehara Y. Microsatellite instability in gastrointestinal tract cancers: a brief update. Surg Today 2005; 35: 1005-15. 